\title{
Application of near critical behavior of equilibrium ratios to phase equilibrium calculations
}

\author{
Dan Vladimir Nichita ${ }^{1, *}$, Daniel Broseta ${ }^{1}$, and François Montel ${ }^{2,1}$ \\ ${ }^{1}$ CNRS UMR 5150, Laboratoire des Fluides Complexes et leurs Réservoirs, Université de Pau et des Pays de l'Adour, \\ BP 1155, 64013 Pau Cedex, France \\ ${ }^{2}$ Centre Scientifique et Technique Jean Feger, Total, avenue Larribau, 64018 Pau Cedex, France
}

Received: 14 June 2019 / Accepted: 18 September 2019

\begin{abstract}
We examine the asymptotic behavior of the equilibrium ratios $\left(K_{i}\right)$ near the convergence locus in the pressure-temperature plane. When the Equation of State (EoS) is analytical, which is the case of most EoS of engineering purpose, $K_{i}$ tends towards unity or, equivalently, its $\operatorname{logarithm} \ln K_{i}$ tends to zero, according to a power $1 / 2$ of the distance to this locus. As a consequence, if $\ln K_{i}$ is expressed as a linear combination of pure component parameters with coefficients only depending on mixture phase properties (i.e., reduction parameters), these coefficients obey a similar power law. Deviations from the $1 / 2$ power law are thus fairly limited for $\ln K_{i}$ and for the reduction parameters (at least in the negative flash window between the convergence locus and the phase boundaries), which can be exploited to speed up flash calculations and for quickly determining approximate saturation points and convergence pressures and temperatures. The chosen examples are representative synthetic and natural hydrocarbon mixtures, as well as various injection gas-hydrocarbon systems.
\end{abstract}

\section{List of symbols}

A $\quad$ EoS parameter

$A_{i} \quad$ Component parameter in the EoS

a EoS parameter

$B \quad$ EoS parameter

$B_{i} \quad$ Component parameter in the EoS

$b \quad$ EoS parameter

$C_{k} \quad$ Coefficients in $\ln K_{\mathrm{i}}$ expression

$f_{i} \quad$ Fugacity of component $i$ in the mixture

$K_{i} \quad$ Equilibrium ratio $\left(=y_{i} / x_{\mathrm{i}}\right)$

$k_{i j} \quad$ Binary Interaction Parameter (BIP) between components $i$ and $j$

$L \quad$ liquid mole fraction

$m \quad$ Number of nonzero BIPs

$m_{k} \quad$ Critical amplitudes

$M \quad$ Number of reduction parameters

$n \quad$ Number of components

$n_{i} \quad$ Number of moles

$P \quad$ Pressure

$\mathrm{q}_{\alpha \mathrm{i}} \quad$ Elements of the reduction matrix

$R \quad$ Universal gas constant

T Temperature

$\begin{array}{ll}V & \text { Vapor mole fraction } \\ x_{i} & \text { Liquid mole fraction, component } i \\ y_{i} & \text { Vapor mole fraction, component } i \\ z_{i} & \text { Feed mole fraction, component } i \\ \mathrm{Z} & \text { Compressibility factor }\end{array}$

Greek letters

$\begin{array}{ll}\alpha & \text { Reduction parameter } \\ \beta & \text { Critical exponent } \\ \gamma & \text { Reduction parameter } \\ \varphi_{\mathrm{i}} & \text { Fugacity coefficient of component } i \text { in a mix- } \\ & \text { ture } \\ \xi=\left(1-P / P_{\text {conv }}\right. & )^{0.5} \\ \theta & \text { Reduction parameter }\end{array}$

Subscripts

$\begin{array}{ll}c & \text { Critical } \\ \text { conv } & \text { Convergence } \\ i, j & \text { Component index } \\ L & \text { Liquid phase } \\ V & \text { Vapor phase } \\ \text { sat } & \text { Saturation }\end{array}$

\footnotetext{
* Corresponding author: dnichita@univ-pau.fr
} 


\section{Superscripts}

T Transposed

* $\quad$ Reference conditions

\section{Introduction}

Flash calculations represent a large fraction of the computer time in the simulation of many industrial processes. This is for instance the case of compositional reservoir simulations, which consist in the simulation of phase and flow behavior of oil and gas through heterogeneous porous media for reservoir engineering purposes (Ben Gharbia and Flauraud, 2019, He et al., 2019, Luo et al., 2019, Montel, 1998). In these simulations, a huge number (up to billions) of flash calculations (one for each grid block representing a homogeneous region of the reservoir) are carried out for multicomponent fluids with a dozen or more components or pseudo-components, and these flash calculations must be repeated for every time step. A flash calculation is an iterative computational process for finding the equilibrium ratios $K_{i}=y_{i} / x_{i}\left(x_{i}\right.$ and $y_{i}$ are the equilibrium compositions of component $i$ in the liquid and vapor phases), starting from "initial" values of the composition ratios $K_{i 0}$. The process is more rapid if the initial guess $K_{i 0}$ are closer to the equilibrium ratios $K_{i}$; high-quality initial estimates are important especially for "difficult" regions of the phase envelope (critical points and convergence points, where the equilibrium ratios $K_{i}$ tend to unity, see next section, as well as the immediate vicinity of phase boundaries). Some compositional reservoir simulators (Wang et al., 1997) take advantage of the so-called "negative flash", as an alternative to phase stability testing.

Two-phase vapor-liquid phase equilibrium calculations can be initialized using the results of phase stability testing (Michelsen, 1982a) or from ideal equilibrium constants; The latter initial estimates are very poor, except at low pressures, while the former require the resolution of a nonlinear system of equations (twice if the trial phase is not known a priori to be vapor or liquid).

One route to obtain "good" values of $K_{i 0}$ is to extrapolate the equilibrium ratios obtained at the previous time step (Mehra et al., 1982; Nghiem and Li, 1990; Nichita et al., 2007a; Wang and Stenby, 1994) at close pressure, temperature and composition. Another possible route, which is pursued in this paper, consists in exploiting a regularity related to the analyticity of the Equation of State (EoS) and thermodynamic potentials: upon approaching a convergence point, these ratios tend to unity according to a simple scaling law - characterized by an exponent $1 / 2$ - of the temperature or pressure. The EoS of concern here are analytical, which is the case of most EoS used for engineering applications.

It appears that there is an increasing interest to implement simplified rapid phase behavior calculations procedures in compositional reservoir modeling, to achieve significant performance improvement without losing prediction accuracy. Gaganis and Varotsis (2014) proposed an integrated approach using automatically generated classification and regression models (fully replacing conventional routines in the simulator) to provide direct answers to both the phase stability and phase split problems; the dimensionality of the model can be lowered by using the reduced variables framework.

Gaganis (2018) presented a new phase stability method applicable when repeated phase behavior calculations are required, based on simple off line generated discriminating functions. The $K$-values based methods (interpolating equilibrium constants as functions of pressure and composition) are widely used in thermal compositional simulation (Zaydullin et al., 2014, 2016). Rannou et al. (2013) used a tie-line-based equilibrium constants method that captures the compositional dependence of the phase behavior.

The outline of the paper is as follows. After reminding of the properties of convergence points and the concept of negative flash, we present the general expressions for the asymptotic behavior of the natural logarithms of equilibrium constants when a convergence point is approached. Several examples are then considered, including synthetic and natural hydrocarbon fluids and their mixtures with injection gases, which are examined by using a general form of two-parameter cubic EoS (given in an Appendix together with the associated reduction parameters).

\section{A reminder on convergence points}

The concept of convergence pressure dates back to the early 1950 's when it was used in conjunction with charts of equilibrium ratios for the determination of equilibrium phase compositions (Kaliappan and Rowe, 1971; Kazemi et al., 1978; Rowe, 1967) until the development in the 1970's of equations of state of engineering purpose and computer-assisted flash calculations. In these calculations, the "initial" $K_{i}$ values required for starting the iterative computational process are an essential input, which is in many cases were obtained by using approximate methods based on the concept of convergence.

For a given multi-component mixture, the convergence pressure (or temperature) is the pressure (or temperature) where the equilibrium ratios of all mixture components converge toward unity when pressure (or temperature) is increased, the other parameter (i.e., temperature or pressure) being fixed. The critical pressure $P_{c}$ or temperature $T_{c}$ are one particular convergence pressure and temperature: at $T=T_{c}$ the equilibrium ratios converge to unity when pressure $P$ is increased and approaches $P_{c}$. For other temperatures $T \neq T_{c}$, the equilibrium ratios converge to unity for a pressure that exceeds the bubble- or dew-point pressure: the convergence pressure $P_{\text {conv }}$ falls in a region in the $T-P$ plane where the fluid is in the single phase state. The interval between the bubble- or dew-point pressure and $P_{\text {conv }}$ corresponds to "negative flashes" (Whitson and Michelsen, 1990): the flash calculation gives a non-trivial solution (the $K_{i}^{\prime}$ s are different from unity) but the liquid and gas mole fractions lie outside the interval of physical solutions $[0,1]$ (the limits of this interval correspond to the bubble- and dew-points). Likewise, for fixed $P$ the convergence temperature $T_{\text {conv }}$ is defined as the temperature 
where the $K_{i}$ 's converge to unity; $T_{\text {conv }}$ lies outside the twophase domain except when $P=P_{c}$ (and then $T_{\text {conv }}=T_{c}$ ). The locus in the $T-P$ plane of convergence pressures and temperatures is termed the Convergence Locus (CL).

The "negative flash" domain is comprised between the CL and the phase boundaries. The mathematical domain of the vapor mole fraction $V$, comprised between two adjacent asymptotes of the Rachford-Rice function $1 /\left[1-\max \left(K_{i}\right)\right]$ and $1 /\left[1-\min \left(K_{i}\right)\right]$, is wider (and in many cases much wider) than the physical interval $V \in[0,1]$. In a negative flash calculation, the vapor (or liquid) mole fraction is allowed to go out of this interval. When a convergence point that is not a critical point is approached, $V \rightarrow \pm \infty$, where the sign depends on which of the two asymptotes is approached (whereas $V \rightarrow 1 / 2$ when a "true" critical point is approached). The main advantage of the negative flash is that all phase properties are continuously derivable at phase boundaries; this property is useful to safely treat phase appearance and disappearance at the crossing of phase boundaries.

On the CL, $K$-values are all equal to unity but component mole fractions $z_{c i}$ are different from feed compositions $z_{i}$, except indeed when the convergence point is a "true" critical point. However, those mole fractions $z_{c i}$ are those of a mixture which has its "true" critical point equal to the convergence point. Moreover, the results of isothermal flashes for $P<P_{\text {conv }}$, or of isobaric flashes for $T<T_{\text {conv }}$, are very close (identical for binary mixtures) for the feed composition $z_{\mathrm{i}}$ and for the "critical composition" $z_{c i}$ (Rowe, 1967) except for the vapor and liquid mole fractions $V$ and $L=1-V$. In the latter case, these fractions remain in the physical domain $[0,1]$ and tend to $1 / 2$ on approach to $P_{\text {conv }}$ or $T_{\text {conv }}$, which are "true" critical pressure or temperature. This feature is exploited in the next section, which goes along with a very peculiar asymptotic behavior of $K_{i}$ 's on approach to a convergence point and several examples will be given in Section 4 .

\section{Asymptotic behavior of equilibrium ratios and their logarithm near convergence points}

The near-critical behavior in the two-phase liquid-vapor region was studied by Dalton and Barieau (1968) and Dalton (1970) for the equilibrium constants of binary mixtures and by Fleming and Vinatieri (1979) in ternary mixtures. It relies on an EoS or a thermodynamic potential that is analytical near the critical point, which allows it to be Taylor-expanded in powers of $\Delta T=T-T_{c}$ or $\Delta P=P-P_{c}$, or of their dimensionless counterparts $\Delta T=\left(T-T_{c}\right) / T_{c}$ and $\Delta P=\left(P-P_{c}\right) / P_{c}$, depending on whether pressure or temperature is varied in the process of interest. The departure from unity of the equilibrium constants turns out to vary according to a "classical" (or mean-field) scaling law as a function of the distance to the critical point, namely $|\Delta T|$ or $|\Delta P|$, with an exponent equal to $1 / 2$. This feature is directly related to the Taylorexpansion (or Landau expansion, Landau and Lifshitz, 1959) of the appropriate thermodynamic potential (in this case the Gibbs free energy) around the critical point (of coordinates $T_{c}$ and $P_{c}$ ), and on vanishing at the critical point of the second- and third-order derivatives of the thermodynamic potential with respect to composition. A similar behavior was proved by Michelsen (1984) for multicomponent mixtures.

The natural logarithms of the equilibrium constants can be approximated as

$$
\ln K_{i} \approx A_{i P}\left(\frac{P_{c}-P}{P_{c}}\right)^{1 / 2}
$$

in the vicinity of the critical point along the critical isotherm, $T=T_{\mathrm{c}}$, in the two-phase region, and

$$
\ln K_{i} \approx A_{i T}\left(\frac{T_{c}-T}{T_{c}}\right)^{1 / 2}
$$

on the critical isobar, $P=P_{c}$, in the two-phase region, where $A_{i P}=f\left(D_{P}, z_{i}, u_{i}\right)$ and $A_{i T}=f\left(D_{T}, z_{i}, u_{i}\right)$ depend on mixture composition, on the eigenvector corresponding to the minimum eigenvalue of the Hessian at the critical point and on third and fourth order partial derivatives of the thermodynamic potential with respect to composition and pressure or temperature. While the calculation of these derivatives is a difficult task and it requires the prior calculation of the critical point it is interesting to note that the critical amplitudes need not be directly calculated; they can be approximated using the information available from a previously performed flash calculation by assuming the $1 / 2$ power law from equations (1) and (2). Note that the equilibrium constants follow the same asymptotic behavior as $\ln K_{i}$ near a critical point (where $K_{i}-1$ are small), but on smaller pressure intervals, since

$$
\ln K_{i}=\ln \left[1+\left(K_{i}-1\right)\right]=K_{i}-1-1 / 2\left(K_{i}-1\right)^{2}+\ldots
$$

At this point it is important to remind that the above mean-field or Landau-type approach provides only an approximation of the near-critical behavior of fluids, which is characterized by scaling exponents that differ from the mean-field (or Landau) values: for instance, the exponent $1 / 2$ in equations (1) and (2) should be replaced by $\beta \approx 0.33$ (for a review see Levelt Sengers et al., 1983). The latter behavior is not considered further in this paper, which is focused on EoS and thermodynamic potentials of engineering purpose, all of which are analytical functions of thermodynamic and composition variables.

Until now, no hypothesis has been made as to the specific analytical EoS or thermodynamic potential used for describing the phase properties of the multi-component mixture. A widely occurring circumstance is when the EoS is a two-parameter cubic EoS, such as the Peng-Robinson (PR) EoS (Peng and Robinson, 1976; Robinson and Peng, 1978), used in next section. In the simplest case where all Binary Interaction Parameters (BIPs) are equal to zero, the equilibrium ratios can be written as (Michelsen, 1986):

$$
\ln K_{i}=C_{0}+C_{1} \sqrt{A_{i}}+C_{2} B_{i}
$$

where $C_{0}, C_{1}$ and $C_{2}$ depend only on bulk quantities (EoS parameters and compressibility factors of both phases at 
given $T, P$ and composition conditions, cf. Appendix) and the coefficients $\sqrt{A_{i}}$ and $B_{\mathrm{i}}$ are related to pure component parameters (see Appendix) and therefore are non singular near the critical point. One may assume that the coefficients $C_{0}, C_{1}$ and $C_{2}$ are exhibiting a similar asymptotical behavior as equilibrium ratios and they are obeying the same scaling law, i.e.,

$$
C_{k}=m_{k}\left(P_{c}-P\right)^{1 / 2} ; \quad k=0,1,2,
$$

where the prefactors (critical amplitudes) $m_{k}$ depend on the fluid system and the EoS used. As will be seen later, the above assumption was confirmed by all examples examined in this paper (as well as in many others not reported here). This feature can be extended to twoparameter cubic EoS with non-zero BIPs (hereafter denoted $k_{i j}$ ) provided $\ln K_{i}$ is properly decomposed (i.e., the mixing rules must be linear forms or decomposable into linear forms). One example (Nichita and Minescu, 2004) is given by the reduction parameters $\gamma_{k i}=\sqrt{A_{i}\left(k_{k i}\right)}(k=1, \ldots, m ; i=k+1, \ldots, n$ : the first $m$ components have nonzero BIPs with the remaining ones) such that:

$$
\ln K_{i}=C_{0}+C_{1} \sqrt{A_{i}}+C_{2} B_{i}+\sum_{k=3}^{m+2} C_{k} \gamma_{k i}
$$

where the term under summation is for the first $m$ components having nonzero BIPs with the remaining ones. In this case there are $m$ additional coefficients $C_{k}$, and equation (5) can be rewritten in the more compact form,

$$
\ln K_{i}=\sum_{k=0}^{m+2} C_{k} q_{k i} ; \quad i=1, \ldots, n,
$$

where $q_{k i} \quad\left(q_{0 i}=1, q_{1 i}=\sqrt{A_{i}}, \quad q_{2 i}=B_{i}, \quad\right.$ and $q_{k i}=\sqrt{A_{i}}\left(1-k_{k i}\right) ; k=3, \ldots, m+2$, for $\left.i=1, \ldots, n\right)$ are the elements of the reduction matrix (Hendriks, 1988), and the coefficients $C_{k}$ depend only on the reduction parameters (directly and via the compressibility factors). Expressions for the coefficients $C_{k}$ are given in the Appendix. Equation (6) remains valid for any reduction procedure (Hendriks and van Bergen, 1992; Nichita, 2006; Nichita and Minescu, 2004), provided appropriate elements of the reduction matrix are used. It is worth noting that equation (6) is a key equation for flash calculations using reduction methods (Nichita and Graciaa, 2011; Petitfrere and Nichita, 2015) and for pseudo-component delumping (Nichita and Leibovici, 2006). Expressions for the coefficients $C_{k}(k>2)$ are given in the Appendix.

As emphasized in the previous section, the results of an isothermal flash (i.e., the equilibrium constants) performed for the fluid composition $z_{i}$ and for the critical composition $z_{i c}$ (corresponding to the current temperature) are very close, therefore equation (1) holds when $P_{c}$ is replaced by $P_{\text {conv }}$ and $z_{i}$ is replaced $z_{i c}$. More generally, any fluid system that is described by an analytical
EoS or thermodynamic potential behaves similarly when approaching a critical (or convergence) point from the two-phase (i.e., liquid - vapor) region: the equilibrium ratios $K_{i}$ tend asymptotically to unity (or, equivalently, $\ln K_{i}$ tend to 0$)$ according to the "universal" scaling law $\left(T_{\text {conv }}-T\right)^{1 / 2}$ or $\left(P_{\text {conv }}-P\right)^{1 / 2}$, depending on whether the critical (or convergence) point is approached by varying the temperature (at constant pressure) or pressure (at constant temperature).

It must be noted that the conditions near the convergence locus are extremely difficult ones for flash calculations. From a computational point of view, a negative flash near the convergence locus is even more difficult than a regular flash near critical points, since the negative flash solution corresponds to a saddle point of the Gibbs free energy hypersurface, while at two-phase conditions the solution is at the global minimum of the Gibbs free energy $G$. The Successive Substitution Iterations (SSIs) scheme only guarantees the convergence to a local minimum of $G$ (Michelsen, 1982b), and at certain conditions in the negative flash domain the SSI method converges to the trivial minimum of the $G$ surface (a more detailed explanation can be found in Whitson and Michelsen, 1990). Thousands of successive substitution iterations may in fact be needed to ensure convergence, and more efficient methods are needed to estimate the convergence pressure without repeatedly performing flash calculations under very difficult conditions.

In the following examples, the negative flash routine and the algorithm for convergence pressure calculation presented by Nichita et al. (2007b) are used in the "exact" flash calculations. A general form of two-parameter cubic EoS is used (see Appendix), and numerical results are obtained by using the Peng-Robinson EoS. Using the reduction parameters limits the scope of the paper to cubic EoS (the most widely used in petroleum engineering). However, a similar methodology can be used for any EoS by exploiting the quasi-linearity of $\ln K_{i}$ (on narrower intervals than in the case of reduction parameters, as will be shown in the next section).

\section{Numerical procedure}

Surprisingly, as will be seen below from numerical examples, little deviations from the asymptotic behavior, equations (1) and (4), are generally observed in the entire negative flash region (between the CL and the saturation curve), and also inside the two-phase region near phase boundaries where flash calculations are usually more difficult (as compared to flashes well inside the two-phase region). These equations can be exploited in various manners that are illustrated in the next section with particular fluid examples. First, it can be exploited to set up a fast algorithm (as compared to the existing ones, Jensen and Michelsen, 1990; Nichita et al., 2007b) for approximate calculations of convergence pressure. Second, this property can be used to extrapolate the results of previously performed flash calculations and provide a good initialization for a negative flash or a two-phase flash near a phase 
boundary. Third, it can be used to inter- and extra-polate $K$-values from known values. Fourth, it allows a rapid approximate determination of saturation pressures.

Another advantage of this formulation is that precalculated tables of $K$-values are not required, because by using equations (4) and (5) only very limited storage is required (essentially only the slopes $m_{k}$ in equation (4) must be stored for given temperature and composition conditions).

Introducing the notation

$$
\xi(p)=\left(\frac{P_{\text {conv }}-P}{P_{\text {conv }}}\right)^{0.5},
$$

the equation of a straight line between a certain reference pressure, $P^{*}$ (where the results of a negative or two-phase flash calculation are available), and $P_{\text {conv }}$ is

$$
C_{k}=C_{k}(P *)+\left.\left(\frac{\partial C_{k}}{\partial \xi}\right)_{T}\right|_{P *}[\xi(P)-\xi(P *)]
$$

with

$$
\frac{\partial C_{k}}{\partial \xi}=\frac{\partial C_{k}}{\partial P} \frac{\mathrm{d} P}{\mathrm{~d} \xi} \quad k=0, \ldots, m+2,
$$

where $\mathrm{d} P / \mathrm{d} \xi=-2 \xi P_{\text {conv }}$, since $P=P_{\text {conv }}\left(1-\xi^{2}\right)$.

At $P=P_{\text {conv }}$, equation (8) reads

$$
0=C_{k}(P *)-\left.\left(\frac{\partial C_{k}}{\partial \xi}\right)_{T}\right|_{P *} \xi(P *) .
$$

The partial derivatives $\left(\partial C_{k} / \partial P\right)_{T}$ have rather simple expressions, and are easily calculated analytically (as described by Nichita, 2008); in fact, in practice it suffices to calculate only one of these partial derivatives, say that of $C_{0}$ (note that its expression does not depend on the number of non-zero BIPs).

The convergence pressure (its approximation considering a linear variation) is obtained from equations (9) and (11) written for $k=0$ :

$$
P_{\text {conv }}=P *-\frac{1}{2} \frac{C_{0}(P *)}{\left.\left(\frac{\partial C_{0}}{\partial P}\right)_{T}\right|_{P *}} .
$$

A similar equation written in terms of temperature can be used for an approximate convergence temperature calculation at given pressure.

Once $P_{\text {conv }}$ is calculated, the amplitudes $m_{k}$ (slopes) are obtained from

$$
m_{k}=C_{k}(P *) / \xi(P *),
$$

then the coefficients $C_{k}$ can be calculated as functions of pressure from

$$
C_{k}(P)=m_{k} \xi(P) ; \quad k=0, \ldots, m+2 ;
$$

and finally the dependences $K_{i}(P)$ are obtained from equation (6) for $i=1, \ldots, n$.

If the results of a flash calculation at a certain pressure (which can be in the two-phase region or in the negative flash region) are available, one can rapidly estimate the phase boundary location. Using $K$-values estimated from equation (6), saturation pressure can be rapidly approximated. Few iterations are required until the dewpoint locus equation $\left(\sum_{i=1}^{n} z_{i} / K_{i}=1\right)$ is satisfied (on the bubble point side, the equation is $\left.\sum_{i=1}^{n} z_{i} K_{i}=1\right)$. A numerical application will be presented in the next section.

The new regularity can also be used to efficiently generate $K$-values tables (some compositional simulators have an option for using $K$-values tables for flash calculations). For domains where the coefficients $C_{k}$ are non-linear, a procedure similar to that presented by Chien and Lee (1983) for $K$-values can be set up. Note that $C_{k}$ generally have a "smoother" variation with pressure than $K$-values, and less storage is required.

\section{Results}

The linear dependence of the $K$-values and of $C_{k}$ with $\left(P_{c}-P\right)^{1 / 2}$, which we call hereafter quasi-linearity, is tested for a model gas-condensate system (Y8) taken from the literature (Yarborough, 1972), and for mixtures of this gas-condensate with nitrogen $\left(\mathrm{Y} 8 / \mathrm{N}_{2}\right)$ : our purpose is to study the influence of an injection gas on convergence pressures and $K$-values in the negative flash region. Then, a reservoir fluid and its mixtures with different amounts of carbon dioxide are studied. The PR EoS is used in all calculations.

\subsection{Y8 synthetic mixture}

Test calculations have been performed first on a synthetic mixture of six normal-alkanes, known in the literature as the Y8 mixture (Yarborough, 1972). Mixture composition and component properties are given in Table 1. All BIPs are set to zero in the PR EoS. Figure 1 depicts the phase envelope and the convergence locus (calculated with the procedure described in Nichita et al., 2007b) of this mixture.

The calculated critical point is $T_{c}=293.78 \mathrm{~K}$ and $P_{c}=210.66$ bar. Plots of equilibrium ratios $K_{i}$ and of their logarithms $\ln K_{i}$ vs. $\left(1-P / P_{c}\right)^{0.5}$ on the critical isotherm (indeed at $T=T_{c}$ the critical pressure is a convergence pressure) are presented in Figures $2 \mathrm{a}$ and $2 \mathrm{~b}$, respectively. As expected from a general argument (see Sect. 3, Eq. (1)), $K_{i}$ tends to unity and $\ln K_{i}$ tends to zero quasilinearly when pressure approaches the critical pressure. Interestingly, $\ln K_{i}$ obeys this quasi-linear behavior and it appears to be closer to linearity in a much larger pressure interval than does $K_{i}$ (it can be observed in Fig. 2b for the two heaviest components that $\ln K_{i}$ behave almost linearly, while an important curvature can be clearly observed in Fig. 2a for these $K_{i}$ on the same interval). The same trends are observed along the critical isobar for the equilibrium ratios $K_{i}$ (Fig. 3a) and for the logarithms $\ln K_{i}$ (Fig. 3b) vs. $\left(1-T / T_{\mathrm{c}}\right)^{0.5}$. The coefficients $C_{k}$ $(k=0,1,2)$ also vary linearly with $\left(1-P / P_{\mathrm{c}}\right)^{0.5}$ at 
Table 1. Composition and component properties of the Y8 mixture.

\begin{tabular}{lcclc}
\hline Component & $z_{i}$ & $T_{c i}, \mathrm{~K}$ & $P_{c i}$, bar & $\omega_{i}$ \\
\hline $\mathrm{C}_{1}$ & 0.8097 & 190.56 & 45.99 & 0.011 \\
$\mathrm{C}_{2}$ & 0.0566 & 305.32 & 48.72 & 0.099 \\
$\mathrm{C}_{3}$ & 0.0306 & 369.83 & 42.48 & 0.152 \\
$n \mathrm{C}_{5}$ & 0.0457 & 469.70 & 33.70 & 0.252 \\
$n \mathrm{C}_{7}$ & 0.0330 & 540.20 & 27.40 & 0.350 \\
$n \mathrm{C}_{10}$ & 0.0244 & 617.70 & 21.10 & 0.490 \\
\hline
\end{tabular}

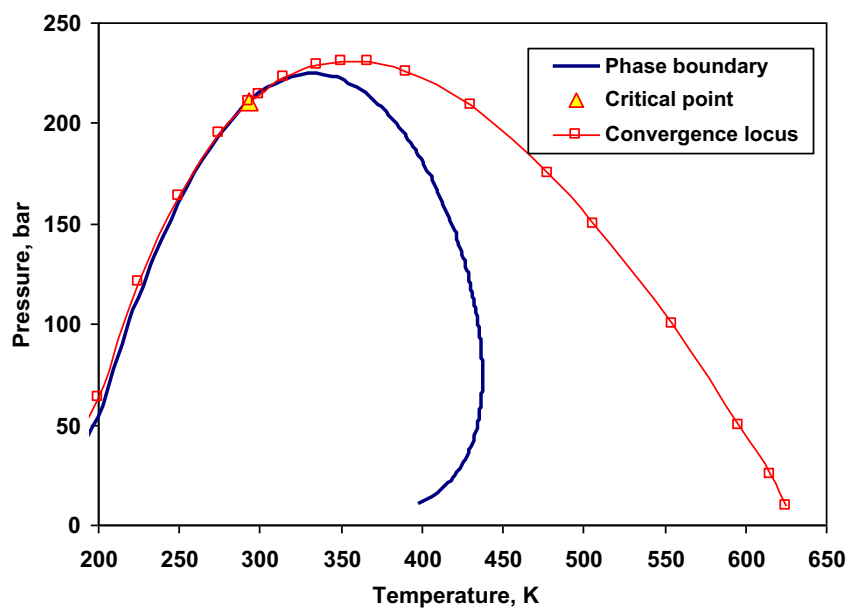

Fig. 1. Phase envelope and convergence locus of the Y8 mixture.

$T=T_{c}$ (Fig. 4a) and with $\left(1-T / T_{c}\right)^{0.5}$ at $P=P_{c}$ (Fig. 4b) over a large pressure or temperature interval (comparable to that of quasi-linear behavior for $\ln K_{i}$ ): the coefficients of determination for the three coefficients are in both cases $R^{2}>0.999$ over the intervals represented in Figures $4 \mathrm{a}$ and $4 \mathrm{~b}$, about $10 \mathrm{bar}$ and $10 \mathrm{~K}$, respectively.

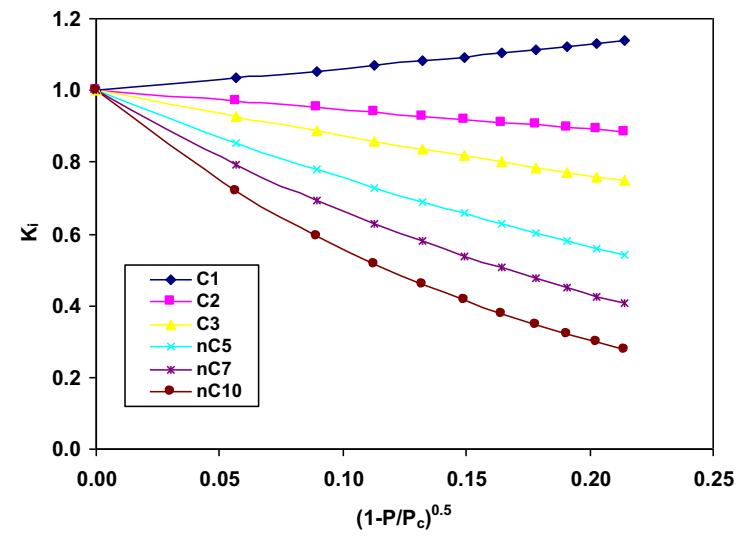

(a)
Let us now analyze the same behavior for a temperature $T \neq T_{c}$. On the isotherm of $T=335 \mathrm{~K}$ (in the region where retrograde condensation occurs), the $K$-values (Fig. 5) and coefficients $C_{k}$ (Fig. 6) are plotted vs. pressure up to the convergence pressure. Note again the quasi-linear behavior for a pressure range of about $10 \mathrm{bar}$. At $T=335 \mathrm{~K}$, the saturation (dew point) pressure is $P_{\text {sat }}=224.39$ bar, and the convergence pressure is $P_{\text {conv }}=229.09$ bar. At $P_{\text {sat }}$, the value of the first coefficient is $C_{0}=0.23876$, and its derivative with respect to pressure is $\left(\partial C_{0} / \partial P\right)_{T}^{\text {sat }}=$ -0.025 . From equation (11), taking $P^{*}=P_{\text {sat }}$, an excellent approximation of the convergence pressure is obtained: $P_{\text {conv }}=229.16$ bar $(0.07$ bar absolute error; the relative error is about $0.03 \%$ ). Note that similar results are obtained using $\left(\partial C_{0} / \partial P\right)_{T}$ at any pressure between $P_{\text {sat }}$ and $P_{\text {conv }}$, and for a certain pressure interval inside the two-phase domain. Convergence pressures have been calculated using this approximation scheme for the entire temperature range up to the cricondentherm (where the difference between convergence and saturation pressure is of the order of 100 bar); the results turn out to be very close to the exact ones (at the scale of Fig. 1, the approximated CL turns out to be undistinguishable from the exact CL).

Suppose a flash calculation is needed at $T=335 \mathrm{~K}$ and $P=215$ bar. One can use as initial guess the $K$-values from a phase stability calculation, or those obtained by an extrapolation technique based on previous flash calculations at some reference conditions. Table 2 lists the exact $K$-values (flash results), those obtained in this work, and those obtained using two extrapolation techniques: the Approximate Flash Calculations (AFC) proposed by Nghiem and Li (1990) and Direct Flash Calculations (DFC) of Wang and Stenby (1994) (earlier suggested by Mehra et al., 1982; it appears to be the most widely used), for a reference pressure $P^{*}=P_{\text {sat }}$. The $K$-values obtained by phase stability testing are also listed, as well as those at the dew point (which give a reasonable initialization for these conditions) and those calculated from the widely-used Wilson (1969) ideal $K$-values relationship (which are poor initial estimates for these conditions). It should be noted that AFC is an iterative method

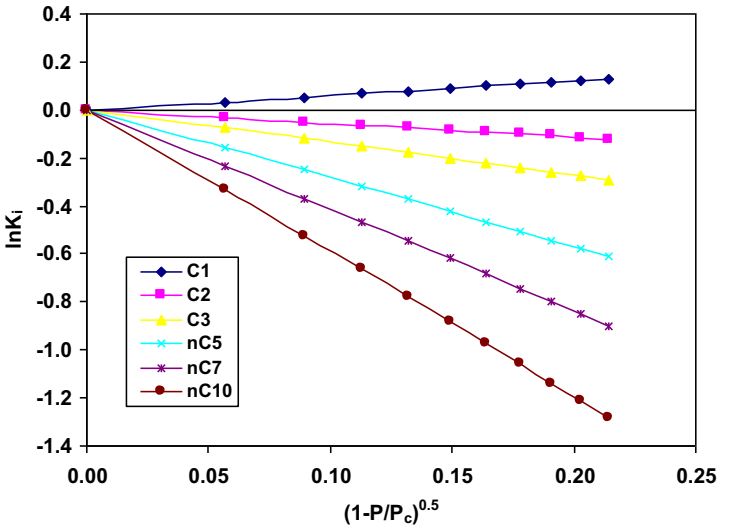

(b)

Fig. 2. (a) Equilibrium ratios vs. $\left(1-P / P_{\mathrm{c}}\right)^{0.5}$ on the critical isotherm $(T=293.78 \mathrm{~K})$ for the Y8 mixture. (b) Logarithm of equilibrium ratios vs. $\left(1-P / P_{\mathrm{c}}\right)^{0.5}$ on the critical isotherm $(T=293.78 \mathrm{~K})$ for the Y8 mixture. 


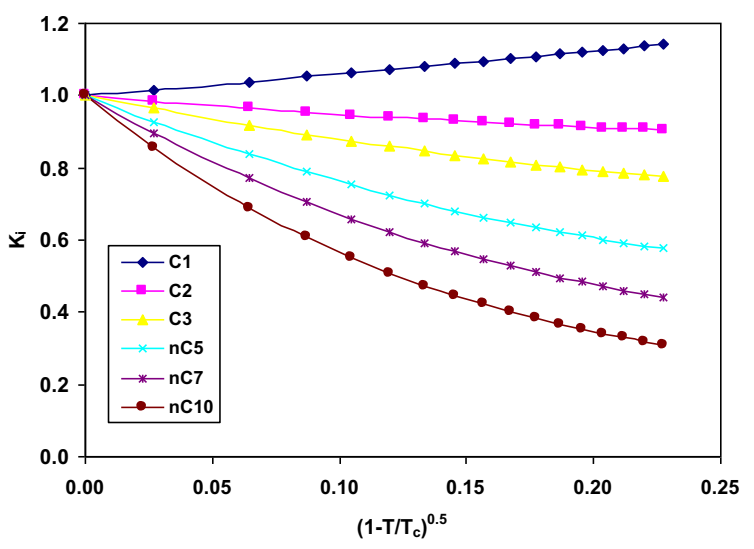

(a)

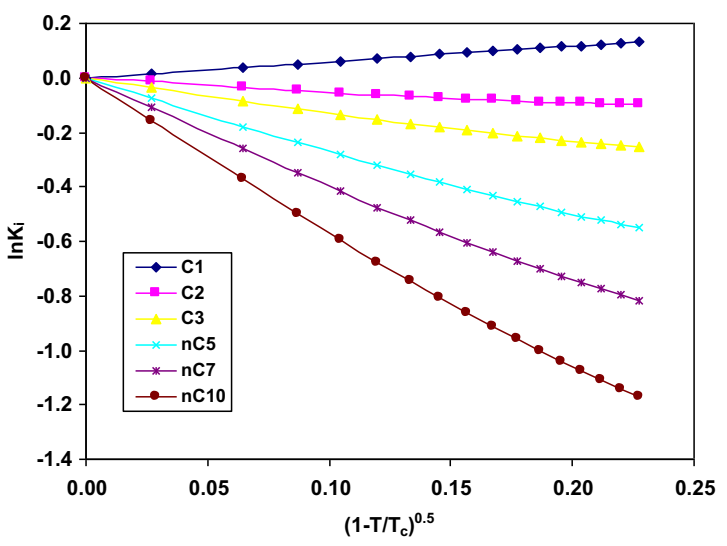

(b)

Fig. 3. (a) Equilibrium ratios vs. $\left(1-T / T_{c}\right)^{0.5}$ on the critical isobar $(P=210.66$ bar $)$ for the Y 8 mixture. (b) Logarithm of equilibrium ratios vs. $\left(1-T / T_{c}\right)^{0.5}$ on the critical isobar $(P=210.66$ bar $)$ for the Y8 mixture.

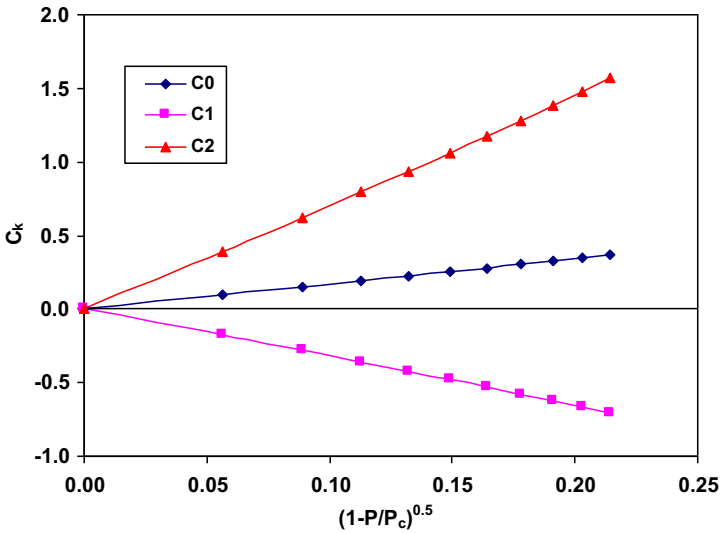

(a)

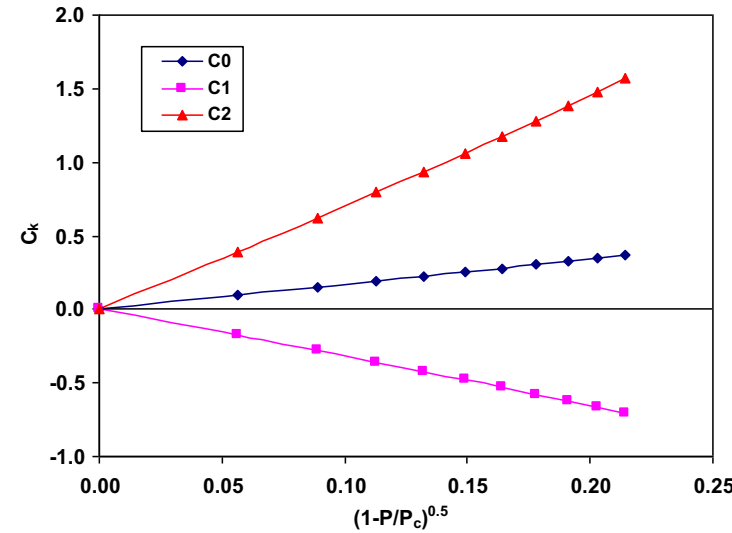

(b)

Fig. 4. (a) Coefficients $C_{k}(k=0,1,2)$ vs. $\left(1-P / P_{c}\right)^{0.5}$ on the critical isotherm $(T=293.78 \mathrm{~K})$ for the Y8 mixture. (b) Coefficients $C_{k}(k=0,1,2)$ vs. $\left(1-T / T_{c}\right)^{0.5}$ on the critical isobar $(P=210.66$ bar $)$ for the Y 8 mixture.

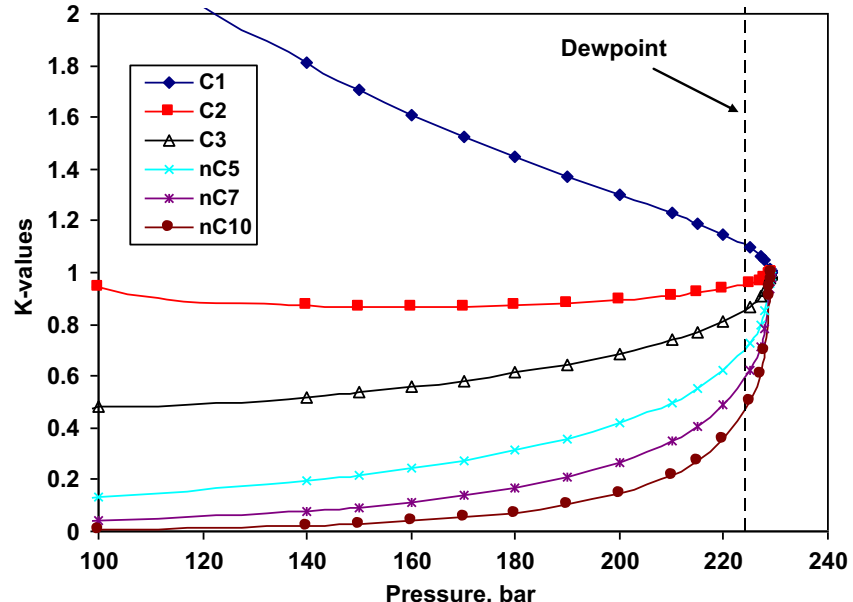

Fig. 5. $K$-values vs. pressure for the $\mathrm{Y} 8$ mixture at $T=335 \mathrm{~K}$.

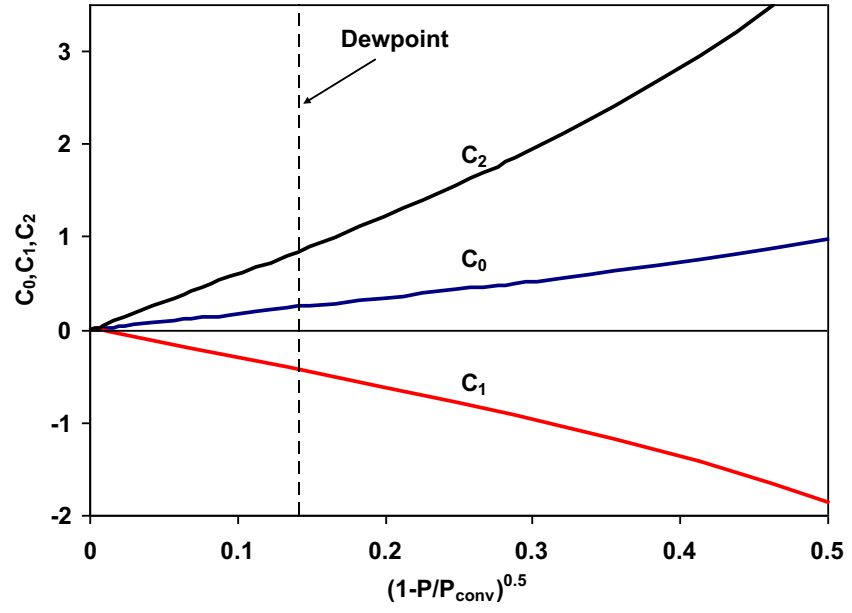

Fig. 6. Coefficients $C_{k}$ vs. $\left(1-P / P_{\text {conv }}\right)^{0.5}$ for the $\mathrm{Y} 8$ mixture at $T=335 \mathrm{~K}$. 
Table 2. Exact flash results and different initial guess for $K$-values for $\mathrm{Y} 8$ mixture at $T=335 \mathrm{~K}$ and $P=215$ bar (the reference pressure is $P^{*}=P_{\text {sat }}=224.39$ bar).

\begin{tabular}{lccccccc}
\hline \multirow{2}{*}{ Component } & \multicolumn{7}{c}{$K$-values } \\
\cline { 2 - 8 } & Exact & This work & AFC & DFC & Stability & Dewpoint & Wilson \\
\hline $\mathrm{C}_{1}$ & 1.190141 & 1.179989 & 1.183721 & 1.169796 & 1.198741 & 1.094757 & 2.232659 \\
$\mathrm{C}_{2}$ & 0.920869 & 0.919780 & 0.922241 & 0.921095 & 0.942890 & 0.955593 & 0.383059 \\
$\mathrm{C}_{3}$ & 0.771288 & 0.773384 & 0.776352 & 0.769698 & 0.801475 & 0.870171 & 0.103682 \\
$n \mathrm{C}_{5}$ & 0.551315 & 0.556511 & 0.560370 & 0.521701 & 0.590329 & 0.728871 & 0.010530 \\
$n \mathrm{C}_{7}$ & 0.406911 & 0.412669 & 0.417393 & 0.334943 & 0.449404 & 0.621142 & 0.001488 \\
$n \mathrm{C}_{10}$ & 0.270341 & 0.275270 & 0.280892 & 0.129642 & 0.313284 & 0.501099 & 0.000116 \\
\hline
\end{tabular}

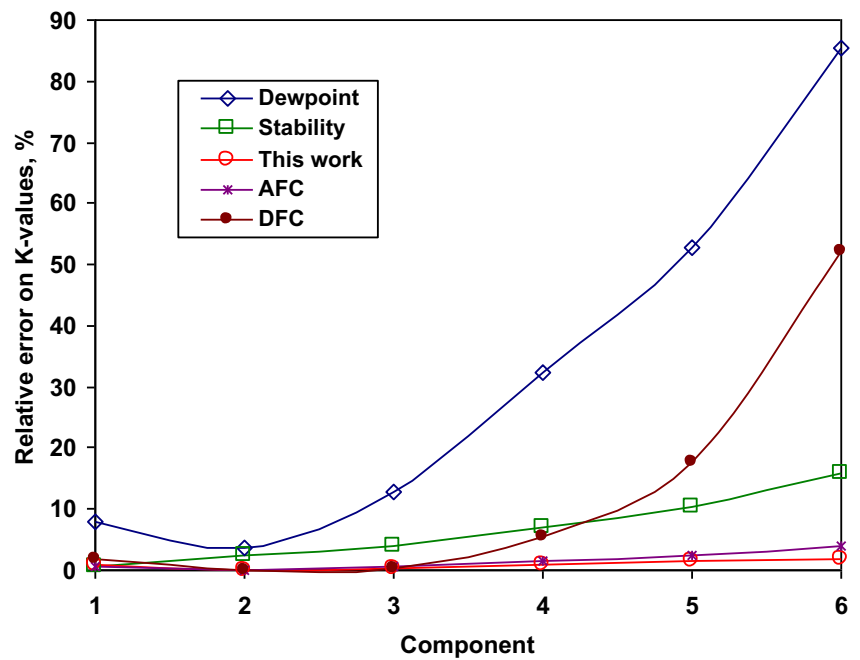

Fig. 7. Relative errors on $K$-values for different (various) initializations. Y8 mixture, flash at $T=335 \mathrm{~K}$ and $P=215$ bar.

(it solves a non-linear system with the Jacobian matrix available from the reference conditions; for this specific calculation it requires six iterations) and DFC requires the resolution of a linear system of equations (also using the Jacobian matrix at the reference conditions); phase stability testing requires six Newton iterations using reduced variables (Nichita and Petitfrere, 2013). The third column in Table 1 gives the $K$-values calculated from equations (11), (12) and (6), using the approximate value of the convergence pressure calculated above. Obviously, the method proposed here provides the best initial guess for $K$-values; the relative errors in $K$-values for each component are given for comparison in Figure 7. In fact, excellent approximations of $K$-values can be obtained for a wide pressure interval.

In practice the difference between current and reference pressure are smaller (in compositional reservoir simulators, the pressure step is bounded via the time step restriction imposed by the stability requirements of the iterative procedure for solving the non-linear system of flow equations); the above example was chosen to enlighten the capabilities of the proposed method.

Suppose now we have the results of a flash calculation at a certain pressure (which can be in the two-phase region

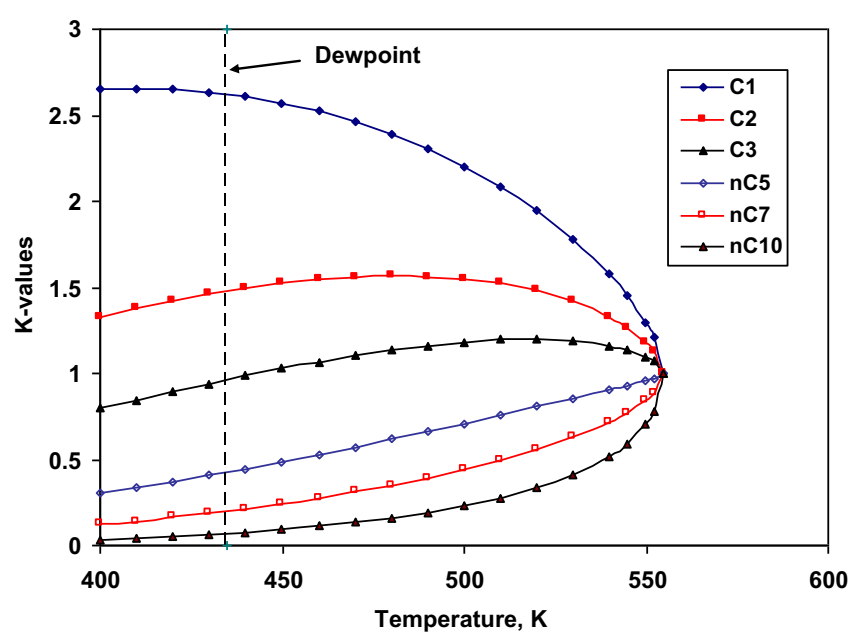

Fig. 8. $K$-values vs. temperature for the $\mathrm{Y} 8$ mixture at $P=100$ bar.

or in the negative flash region) and we want a rapid estimate of the phase boundary location. For example, using $P^{*}=215$ bar (two-phase flash), we obtain $P_{\text {sat }}=$ 224.36 bar, which is very close to the exact one.

Let us now look at what's happening at isobaric conditions. The plot of $K$-values (Fig. 8) and coefficients $C_{k}$ (Fig. 9) vs. $\left(1-T / T_{\text {conv }}\right)^{0.5}$ at $P=100$ bar shows a similar behavior ( with $T_{\text {conv }}=554.32 \mathrm{~K}$ and $T_{\text {sat }}=435 \mathrm{~K}$ ). Note that in this case the linearity holds for hundreds of $K$. A similar method can be readily set up to approximate the convergence temperature at given pressure.

\subsection{Y8/Nitrogen mixtures}

In order to investigate the case of non-zero BIPs, different amounts of nitrogen are combined with the Y8 mixture. The BIPs between $\mathrm{N}_{2}$ and hydrocarbon components are taken equal to $0.1(m=1)$. For a mixture made up of the Y8 fluid $+25 \%$ moles $\mathrm{N}_{2}$, the phase envelope and the convergence locus are plotted in Figure 10. The coefficients $C_{k}$ (Fig. 11) are plotted vs. pressure at $T=300 \mathrm{~K}$. Note that in this case we have four coefficients $C_{k}$, and their linearity holds over the entire negative flash domain. This observation is important because in the description of 


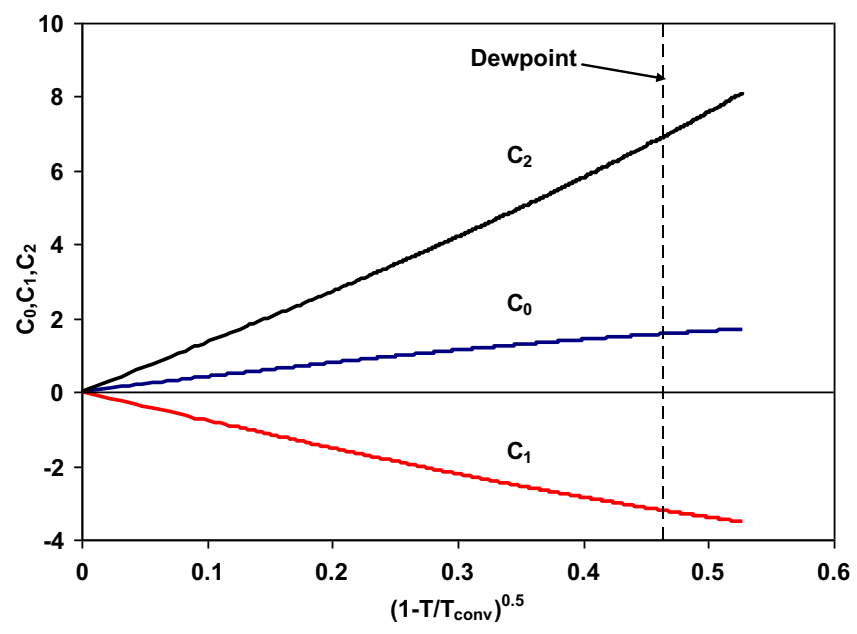

Fig. 9. Coefficients $C_{k}$ vs. $\left(1-T / T_{\text {conv }}\right)^{0.5}$ for the Y 8 mixture at $P=100$ bar.

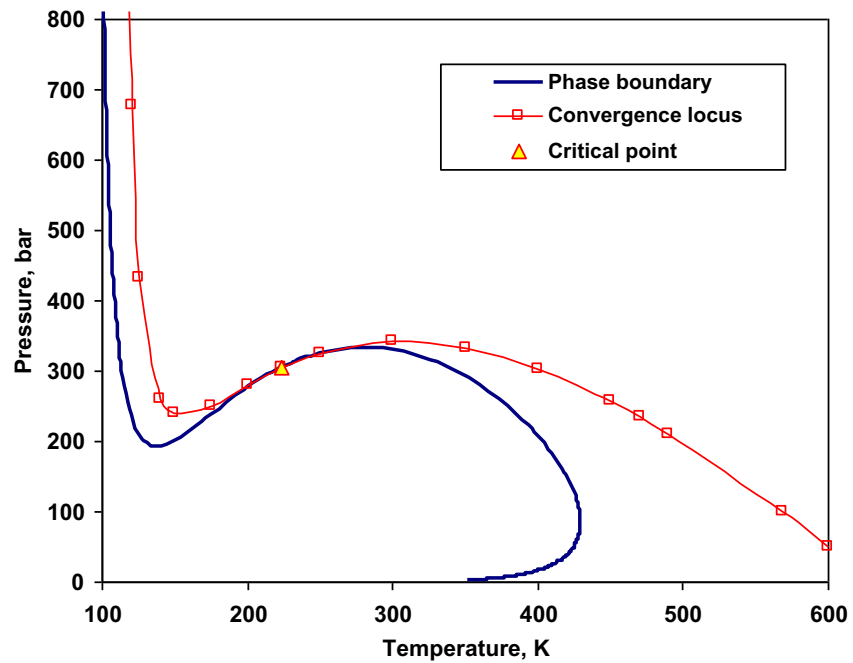

Fig. 10. Phase envelope and convergence locus of $\mathrm{Y} 8+25 \% \mathrm{~N}_{2}$ mixture.

reservoir fluids by cubic EoS non-zero BIPs are usually required. The results of $\mathrm{CL}$ calculations with the exact (Nichita et al., 2007b) and approximate (this work) methods are very close. For a mixture made up of the Y8 fluid $+50 \%$ moles $\mathrm{N}_{2}$, the phase envelope and the convergence locus are plotted in Figure 12 and the coefficients $C_{k}$ are plotted $v s$. pressure at $T=300 \mathrm{~K}$ in Figure 13 . Note that this mixture has no critical points.

\subsection{Reservoir fluid}

The reservoir fluid composition is described by 29 components; eight components have non-zero BIPs with the remaining ones $(m=8)$. Mixture composition and component properties are given in Table 3 and the non-zero BIPs are listed in Table 4 (only elements in the lower triangular part of the symmetric BIPs matrix are given). The PR EoS

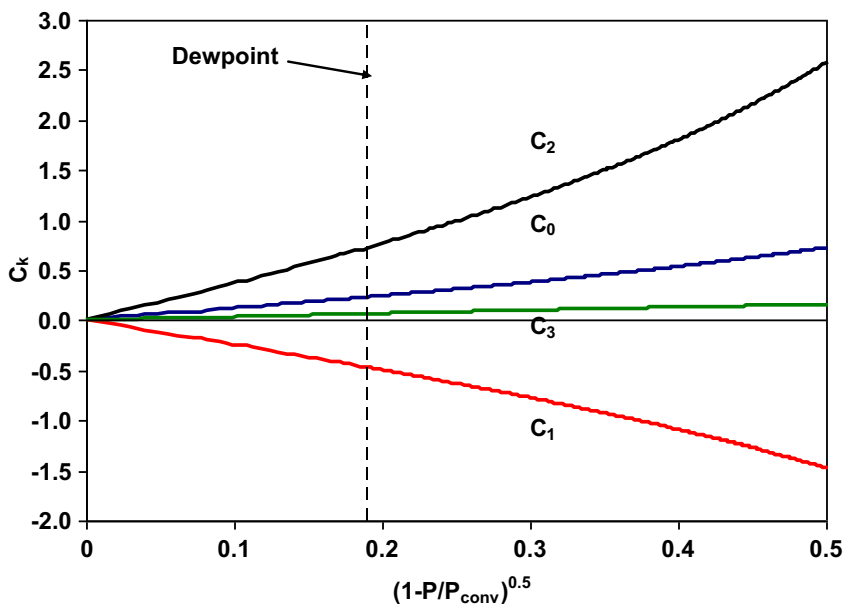

Fig. 11. Coefficients $C_{k}$ vs. $\left(1-P / P_{\text {conv }}\right)^{0.5}$ for the $\mathrm{Y} 8+25 \%$ $\mathrm{N}_{2}$ mixture at $T=300 \mathrm{~K}$.

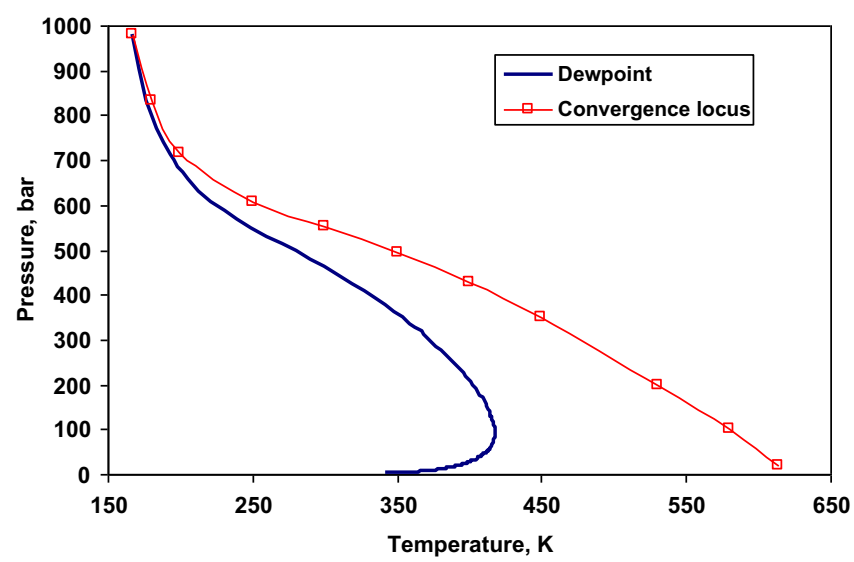

Fig. 12. Phase envelope and convergence locus of $\mathrm{Y} 8+50 \% \mathrm{~N}_{2}$ mixture.

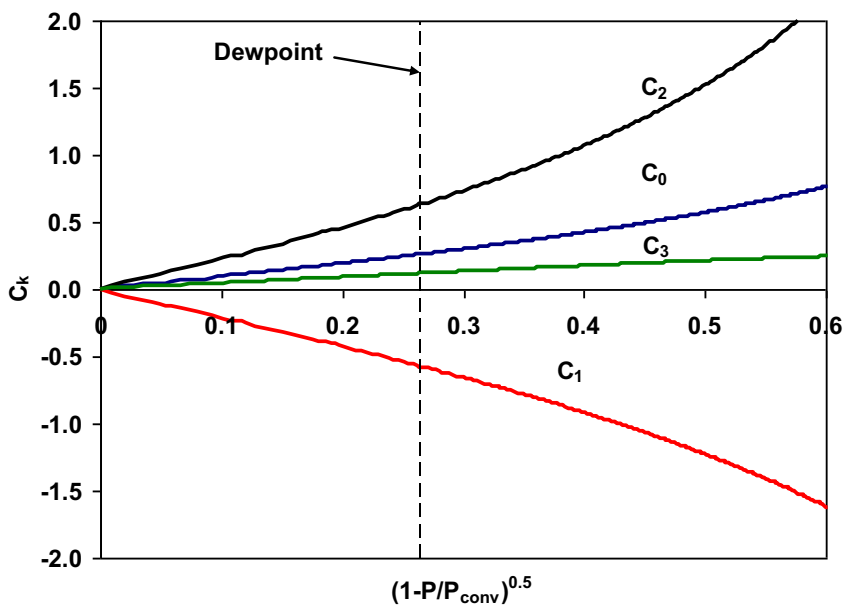

Fig. 13. Coefficients $C_{k}$ vs. $\left(1-P / P_{\text {conv }}\right)^{0.5}$ for the $\mathrm{Y} 8+50 \%$ $\mathrm{N}_{2}$ mixture at $T=300 \mathrm{~K}$. 
Table 3. Reservoir fluid composition and component properties.

\begin{tabular}{lrrllc}
\hline Comp. & $\begin{array}{c}\text { Mole } \\
\text { number }\end{array}$ & MW & $T_{c i}, \mathrm{~K}$ & $P_{c i}$, bar & $\omega_{\mathrm{i}}$ \\
\hline $\mathrm{N}_{2}$ & 1.150 & 28.01 & 126.2 & 33.90 & 0.0400 \\
$\mathrm{CO}_{2}$ & 4.120 & 44.01 & 304.2 & 73.80 & 0.2250 \\
$\mathrm{H}_{2} \mathrm{~S}$ & 14.690 & 34.08 & 373.2 & 89.369 & 0.1000 \\
$\mathrm{CH}_{3} \mathrm{~S}$ & 0.012 & 48.11 & 469.95 & 72.30 & 0.1530 \\
$\mathrm{C}_{2} \mathrm{H}_{6} \mathrm{~S}$ & 0.016 & 62.13 & 499 & 54.90 & 0.1910 \\
$\mathrm{COS}$ & 0.002 & 60.07 & 378.8 & 63.50 & 0.1050 \\
$\mathrm{C}_{1}$ & 48.210 & 16.04 & 190.6 & 46.00 & 0.0115 \\
$\mathrm{C}_{2}$ & 7.320 & 30.07 & 305.4 & 48.80 & 0.0908 \\
$\mathrm{C}_{3}$ & 4.430 & 44.10 & 369.8 & 42.50 & 0.1454 \\
$i \mathrm{C}_{4}$ & 0.860 & 58.12 & 408.1 & 36.50 & 0.1760 \\
$n \mathrm{C}_{4}$ & 1.930 & 58.12 & 425.2 & 38.00 & 0.1928 \\
$i \mathrm{C}_{5}$ & 0.890 & 71.76 & 468.77 & 35.58 & 0.2202 \\
$n \mathrm{C}_{5}$ & 0.880 & 72.15 & 469.6 & 33.70 & 0.2273 \\
$\mathrm{C}_{6}$ & 1.270 & 85.36 & 511.9895 & 33.19 & 0.2606 \\
$\mathrm{BZN}$ & 0.049 & 78.11 & 562.2 & 48.90 & 0.2120 \\
$\mathrm{C}_{7}$ & 1.591 & 99.08 & 549.7539 & 30.63 & 0.2807 \\
$\mathrm{TOL}$ & 0.178 & 92.14 & 591.8 & 41.00 & 0.2630 \\
$\mathrm{C}_{8}$ & 1.702 & 113.24 & 574.715 & 28.30 & 0.3270 \\
$\mathrm{XYL}$ & 0.343 & 106.16 & 621.1333 & 36.00 & 0.3230 \\
$\mathrm{~EB}$ & 0.057 & 106.16 & 617.1 & 36.10 & 0.3011 \\
$\mathrm{C}_{9}$ & 1.250 & 125.19 & 618.15 & 27.58 & 0.3440 \\
$\mathrm{C}_{10}$ & 1.330 & 137.83 & 638.15 & 25.85 & 0.3780 \\
$\mathrm{C}_{11}$ & 1.050 & 149.00 & 658.15 & 24.57 & 0.3960 \\
$\mathrm{C}_{12}$ & 0.840 & 163.00 & 676.15 & 23.20 & 0.4140 \\
$\mathrm{C}_{13}$ & 0.780 & 176.00 & 690.15 & 21.96 & 0.4320 \\
$\mathrm{C}_{14}$ & 0.620 & 191.00 & 708.15 & 20.78 & 0.4590 \\
$\mathrm{CN}_{1}$ & 2.388 & 230.85 & 741.781 & 18.19 & 0.5129 \\
$\mathrm{CN}_{2}$ & 1.762 & 325.39 & 906.15 & 16.80 & 0.6950 \\
$\mathrm{CN}_{3}$ & 0.310 & 500.00 & 1113.15 & 14.35 & 0.8300 \\
\hline & & & & &
\end{tabular}

is used. The phase envelope, the spinodal, and the convergence locus of the reservoir fluid are plotted in Figure 14.

There are $18(2 m+2)$ reduction parameters, and 11 coefficients $C_{k}$ in equation (6). Figure 15 depicts the coefficients $C_{k}$ vs. $\left(1-P / P_{\text {conv }}\right)^{0.5}$ at $T=500 \mathrm{~K}$; at this temperature, there is a wide negative flash region (the dew point pressure is $352 \mathrm{bar}$, and the convergence pressure is 379 bar). As expected, quasi-linearity holds for all coefficients $C_{k}$ over a large pressure interval.

\section{4. $\mathrm{CO}_{2} /$ reservoir fluid mixture}

In order to study the influence of an injection gas on the new regularity, $\mathrm{CO}_{2}$ is added in various amounts to the above reservoir fluid. The phase envelope and the convergence locus for the reservoir fluid $+50 \%$ moles $\mathrm{CO}_{2}$ mixture are presented in Figure 16. Figure 17 plots the coefficients $C_{k}$ vs. $\left(1-P / P_{\text {conv }}\right)^{0.5}$ for the reservoir fluid $+50 \%$ moles $\mathrm{CO}_{2}$ mixture at $T=500 \mathrm{~K}$; some of the $C$ 's (i.e., $C_{0}$,
Table 4. Non-zero BIPS for the reservoir fluid.

\begin{tabular}{|c|c|c|c|c|c|c|c|c|}
\hline Comp. & $\mathrm{N}_{2}$ & $\mathrm{CO}_{2}$ & $\mathrm{H}_{2} \mathrm{~S}$ & $\mathrm{C}_{2} \mathrm{H}_{6} \mathrm{~S}$ & COS & $\mathrm{C}_{1}$ & $\mathrm{C}_{2}$ & $\mathrm{C}_{3}$ \\
\hline \multicolumn{9}{|l|}{$\mathrm{N}_{2}$} \\
\hline $\mathrm{CO}_{2}$ & -0.02 & & & & & & & \\
\hline $\mathrm{H}_{2} \mathrm{~S}$ & 0.18 & 0.1 & & & & & & \\
\hline \multicolumn{9}{|l|}{$\mathrm{CH}_{3} \mathrm{~S}$} \\
\hline \multicolumn{9}{|l|}{$\mathrm{C}_{2} \mathrm{H}_{6} \mathrm{~S}$} \\
\hline \multicolumn{9}{|l|}{ COS } \\
\hline $\mathrm{C}_{1}$ & 0.04 & 0.1 & & 0.15 & & & & \\
\hline $\mathrm{C}_{2}$ & 0.05 & 0.13 & 0.15 & & & & & \\
\hline $\mathrm{C}_{3}$ & 0.08 & 0.13 & 0.09 & & & & & \\
\hline$i \mathrm{C}_{4}$ & 0.1 & 0.13 & 0.075 & & & & & \\
\hline$n \mathrm{C}_{4}$ & 0.09 & 0.13 & 0.05 & & & & & \\
\hline$i \mathrm{C}_{5}$ & 0.1 & 0.12 & 0.06 & & & & & \\
\hline$n \mathrm{C}_{5}$ & 0.1 & 0.12 & 0.06 & & & & & \\
\hline $\mathrm{C}_{6}$ & 0.1 & 0.1 & 0.06 & & 0.1 & & & \\
\hline BZN & 0.1 & 0.1 & 0.06 & & 0.1 & 0.07 & 0.03 & \\
\hline $\mathrm{C}_{7}$ & 0.1 & 0.1 & 0.06 & & 0.1 & & & \\
\hline TOL & 0.1 & 0.1 & 0.06 & & 0.1 & 0.07 & 0.03 & \\
\hline $\mathrm{C}_{8}$ & 0.1 & 0.1 & 0.06 & & 0.1 & & & \\
\hline XYL & 0.1 & 0.1 & 0.06 & & 0.1 & 0.07 & 0.03 & \\
\hline EB & 0.1 & 0.1 & 0.06 & & 0.1 & 0.07 & 0.03 & \\
\hline $\mathrm{C}_{9}$ & 0.1 & 0.1 & 0.06 & & 0.1 & & & \\
\hline $\mathrm{C}_{10}$ & 0.1 & 0.1 & 0.06 & & 0.1 & & & \\
\hline $\mathrm{C}_{11}$ & 0.1 & 0.1 & 0.06 & & 0.1 & & & \\
\hline $\mathrm{C}_{12}$ & 0.1 & 0.1 & 0.06 & & 0.1 & & & \\
\hline $\mathrm{C}_{13}$ & 0.1 & 0.1 & 0.06 & & 0.1 & & & \\
\hline $\mathrm{C}_{14}$ & 0.1 & 0.1 & 0.06 & & 0.1 & & & \\
\hline $\mathrm{CN} 1$ & 0.1 & 0.1 & 0.06 & & 0.1 & 0.02 & & \\
\hline $\mathrm{CN} 2$ & 0.1 & 0.1 & 0.06 & & 0.15 & 0.08 & 0.04 & 0.03 \\
\hline CN3 & 0.1 & 0.1 & 0.06 & & 0.15 & 0.08 & 0.04 & 0.03 \\
\hline
\end{tabular}

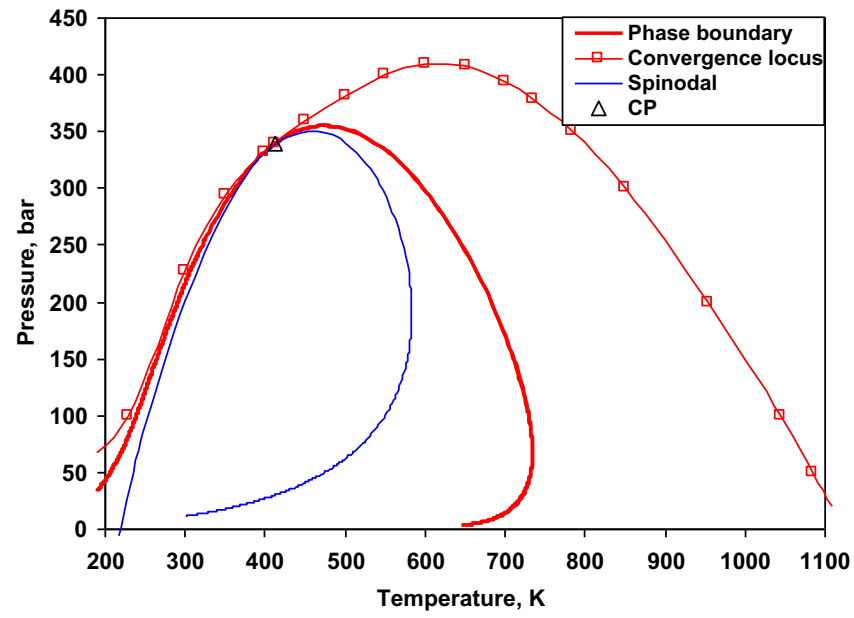

Fig. 14. Phase envelope and convergence locus of the reservoir fluid. 


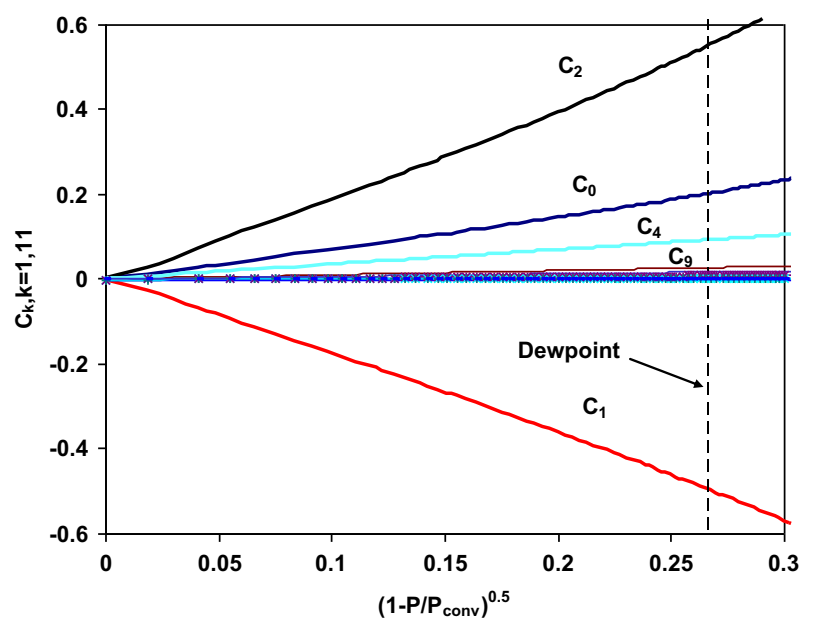

Fig. 15. Coefficients $C_{k}$ vs. $\left(1-P / P_{\text {conv }}\right)^{0.5}$ for the reservoir fluid at $T=500 \mathrm{~K}$.

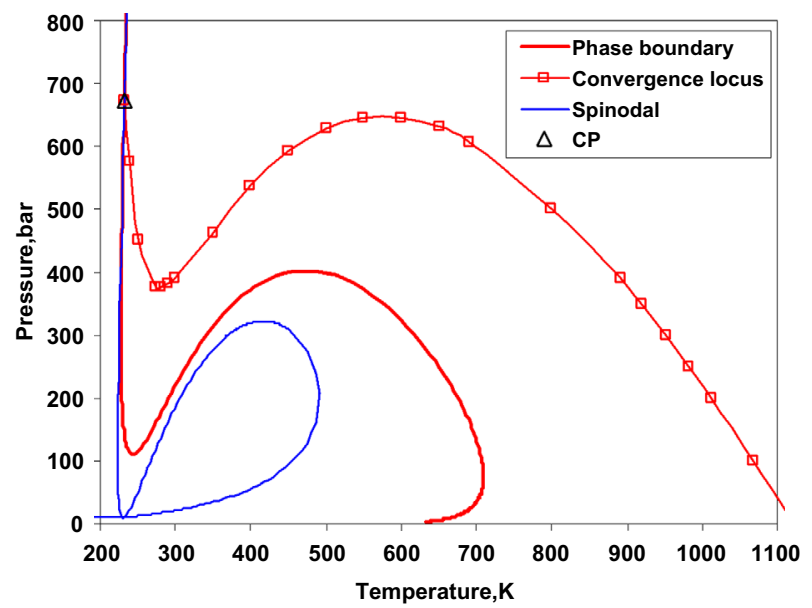

Fig. 16. Phase envelope and convergence locus for the reservoir fluid $+50 \% \mathrm{CO}_{2}$ mixture.

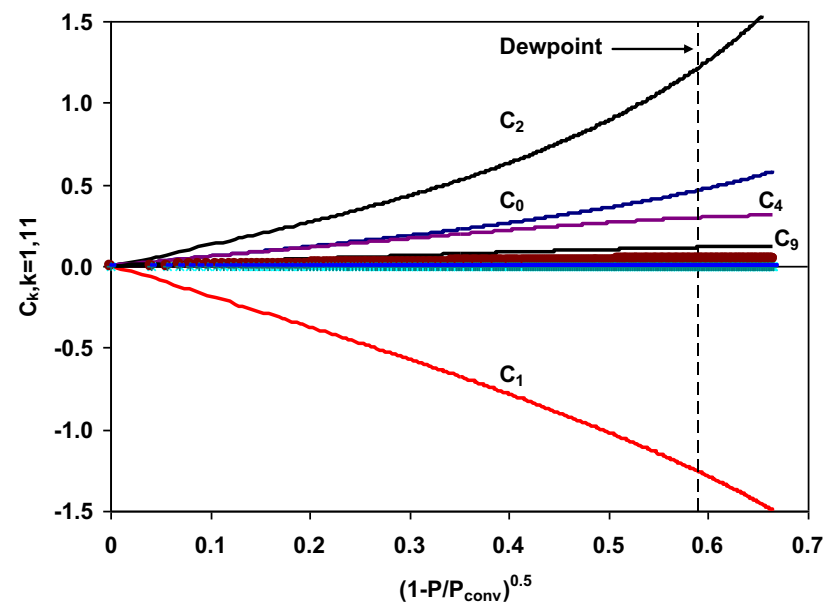

Fig. 17. Coefficients $C_{k}$ vs. $\left(1-P / P_{\text {conv }}\right)^{0.5}$ for the reservoir fluid $+50 \% \mathrm{CO}_{2}$ mixture at $T=500 \mathrm{~K}$.
$C_{1}, C_{2}$ ) are somewhat deviating from linearity, while for the $C$-parameters corresponding to small BIPs deviate from linearity negligibly over a wide pressure range (here to the order of hundreds bar, as the dew point pressure is 398 bar, and the convergence pressure is 628 bar). This example indicates that it is preferable to assume linearity for the latter $C$-parameters.

\section{Conclusion}

The asymptotic behavior of the equilibrium ratios and their logarithm is examined near critical points and convergence points. For any analytical EoS, the $K$-values tend towards unity or, equivalently, their logarithms tend to 0 , according to a power $1 / 2$ of the distance to the critical point or convergence point.

Since the elements of the reduction matrix are never singular at critical points, the coefficients $C_{k}$ for the multilinear expression of the logarithms of $K$-values also behave according to the same power law, which turn out to holds in a larger interval, as observed in a series of fluid examples using the conventional PR EoS. The coefficients $C_{k}$ are frequently observed to behave according to this power law over the entire negative flash region, and a region inside the two-phase pressure/temperature domain (except for systems containing a hydrocarbon mixture and a high concentration of a non-hydrocarbon component, such as carbon dioxide or nitrogen).

This new regularity can be exploited in several ways; it is shown how to rapidly calculate approximate convergence pressures (avoiding costly flash calculations in the vicinity of the convergence locus), extrapolate the results of previously performed flashes to obtain a high quality initial guess for flash calculations, interpolate in rapidly generated $K$-values tables, rapidly approximate phase boundary location. Several numerical examples are given using a cubic EoS and fluid systems (synthetic and naturally occurring) taken from the literature, showing the extrapolating capabilities of the proposed method.

Acknowledgments. We thank Total SA for partial financial support and for the permission to publish this work.

\section{References}

Ben Gharbia I., Flauraud E. (2019) Study of compositional multiphase flow formulation using complementarity conditions, Oil Gas Sci. Technol. - Rev. IFP Energies nouvelles 74, 43.

Chien M.C., Lee S.T. (1983) A new equilibrium coefficient correlation method for compositional simulators, SPE-12243MS, Reservoir Simulation Symposium, November 15-18, San Francisco, CA.

Dalton B.J. (1970) Two-phase equilibria of analytical binary solutions near the critical point, U.S. Bureau of Mines, Information Circular 8486.

Dalton B.J., Barieau R.E. (1968) Equations for calculating various thermodynamic functions of a two-component system from an empirical equation of state, including liquid-vapor equilibria data, Technical Report BM-RI-7076, U.S. Bureau of Mines, Amarillo, TX. 
Fleming P.D. III, Vinatieri J.E. (1979) Quantitative interpretation of phase volume behavior of multicomponent systems near critical points, AIChE J. 25, 493-502.

Gaganis V. (2018) Rapid phase stability calculations in fluid flow simulation using simple discriminating functions, Comput. Chem. Eng. 108, 112-127.

Gaganis V., Varotsis N. (2014) An integrated approach for rapid phase behavior calculations in compositional modeling, $J$. Petrol. Sci. Eng. 118, 74-87.

He C., Mu L., Xu A., Zhao L., He J., Zhang A., Shan F., Luo E. (2019) Phase behavior and miscible mechanism in the displacement of crude oil with associated sour gas, Oil Gas Sci. Technol. - Rev. IFP Energies nouvelles 74, 54.

Hendriks E.M. (1988) Reduction theorem for phase equilibrium problems, Ind. Eng. Chem. Res. 27, 1728-1732.

Hendriks E.M., van Bergen A.R.D. (1992) Application of a reduction method to phase equilibria calculations, Fluid Phase Equilib. 74, 17-34.

Jensen F., Michelsen M.L. (1990) Calculation of first contact and multiple contact minimum miscibility pressure, In Situ 14, 1-17.

Kaliappan C.S., Rowe A.M. (1971) Calculation of pressuretemperature phase envelopes of multicomponent systems, Soc. Petrol. Eng. J. 11, 243-251.

Kazemi H., Vestal C.R., Shank D.G. (1978) An efficient multicomponent numerical simulator, Soc. Petr. Eng. J. 18, 355-368.

Landau L., Lifshitz E. (1959) Fluid mechanics, Pergamon, Section 64

Levelt Sengers J.M.H., Morrison G., Chang R.F. (1983) Critical behavior in fluids and fluid mixtures, Fluid Phase Equilib. 14, 19-44.

Luo E., Fan Z., Hu Y., Zhao L., Wang J. (2019) An evaluation on mechanisms of miscibility development in acid gas injection for volatile oil reservoirs, Oil Gas Sci. Technol. - Rev. IFP Energies nouvelles 74, 59 .

Mehra R.K., Heidemann R.A., Aziz K. (1982) Computation of multiphase equilibrium for compositional simulation, Soc. Petrol. Eng. J 22, 61-68.

Michelsen M.L. (1982a) The isothermal flash problem. Part I. Stability, Fluid Phase Equilib. 9, 1-19.

Michelsen M.L. (1982b) The isothermal flash problem. Part II. Phase split calculation, Fluid Phase Equilib. 9, 21-40.

Michelsen M.L. (1984) Calculation of critical points and phase boundaries in the critical region, Fluid Phase Equilib. 16, 57-76.

Michelsen M.L. (1986) Simplified flash calculations for cubic equations of state, Ind. Eng. Chem. Proc. Des. Dev. 25, 84-188.

Montel F. (1998) New tools for oil and gas reservoir fluid management, Revue de l'Institut Français du Pétrole 53, 9-11.

Nghiem L.X., Li Y.K. (1990) Approximate flash calculations for equation-of-state compositional models, Soc. Petrol. Eng. Res. Eng. 5, 107-114.

Nichita D.V. (2006) a reduction method for phase equilibrium calculation with cubic equations of state, Braz. J. Chem. Eng 23, 427-434.

Nichita D.V. (2008) Phase envelope construction for mixtures with many components, Energy Fuels 22, 488-495.

Nichita D.V., Graciaa A. (2011) A new reduction method for phase equilibrium calculations, Fluid Phase Equilib. 302, $226-233$.
Nichita D.V., Leibovici C.F. (2006) An analytical-component delumping procedure for equations of state with non-zero binary interaction consistent pseudoparameters, Fluid Phase Equilib. 245, 71-82.

Nichita D.V., Minescu F. (2004) Efficient phase equilibrium calculations in a reduced flash context, Can. J. Chem. Eng. 82, $1225-1238$.

Nichita D.V., Petitfrere M. (2013) Phase stability analysis using a reduction method, Fluid Phase Equilib. 358, 27-39.

Nichita D.V., Broseta D., de Hemptinne J.-C., Lachet V. (2007a) Efficient phase equilibrium calculation for compositional simulation: the direct reduced flash, Petrol. Sci. Technol. 25, 315-342.

Nichita D.V., Broseta D., Montel F. (2007b) Calculation of convergence pressure/temperature and stability test limit loci of mixtures with cubic equations of state, Fluid Phase Equilib. 261, 176-184.

Peng D.Y., Robinson D.B. (1976) A new two-constant equation of state, Ind. Eng. Chem. Fundam. 15, 59-64.

Petitfrere M., Nichita D.V. (2015) Multiphase equilibrium calculations using a reduction method, Fluid Phase Equilib. 401, 110-126.

Rannou G., Voskov D., Tchelepi H. (2013) Tie-line-based K-value method for compositional simulation, SPE J. 18, 1112-1122.

Robinson D.B., Peng D.Y. (1978) The characterization of the heptanes and heavier fractions for the GPA Peng-Robinson programs, Research Report, Gas Processors Association, Tulsa, Okla, RR-28.

Rowe A.M. (1967) The critical composition method - a new convergence pressure method, Soc. Petrol. Eng. J. 7, 54-60.

Soave G. (1972) Equilibrium constants from a modified Redlich-Kwong equation of state, Chem. Eng. Sci. 27, 1197-1203.

Wang P., Stenby E.H. (1994) Non-iterative flash calculation algorithm in compositional reservoir simulation, Fluid Phase Equilib. 95, 93-108.

Wang P., Yotov I., Wheeler M., Arbogast T., Dawson C., Parashar M., Sepehrnoori K. (1997) A new generation EOS compositional simulator: Part I - Formulation and Discretization, SPE-37979-MSSPE Reservoir Simulation Symposium, 8-11 June, Dallas, Texas.

Whitson C.H., Michelsen M.L. (1990) The negative flash, Fluid Phase Equilib. 53, 51-72.

Wilson G. (1969) A modified Redlich-Kwong equation of state, application to general physical data calculations, Paper no. 15 C presented at the AIChE 65th National Meeting, May 4-7, Cleveland, Ohio.

Yarborough L. (1972) Vapor-liquid equilibrium data for multicomponent mixtures containing hydrocarbon and non-hydrocarbon components, J. Chem. Eng. Data 17, 129-133.

Zaydullin R., Voskov D., James S.C., Lucia A. (2014) Fully compositional and thermal reservoir simulation, Comput. Chem. Eng. 63, 51-65.

Zaydullin R., Voskov D., Tchelepi H. (2016) Comparison of EoS-based and K-values-based methods for three-phase thermal simulation, Transp. Porous Media 116, 663-686. 


\section{Appendix}

\section{Cubic Equation of State and expressions of the coefficients $C_{k}$}

The general form of two-parameter cubic EoS is

$$
p(T, v)=\frac{R T}{v-b}-\frac{a(T)}{\left(v+\delta_{1} b\right)\left(v+\delta_{2} b\right)} .
$$

Equation (A1) includes the SRK EoS (Soave, 1972, for $\delta_{1}=1$ and $\delta_{2}=0$ ) and the PR EoS (Peng and Robinson, 1976 , for $\left.\boldsymbol{\delta}_{1,2}=\mathbf{1} \pm \sqrt{\mathbf{2}}\right)$. With $Z=p v / R T, A=a p /$ $(R T)^{2}$, and $B=b p / R T$, the implicit form (in the compressibility factor Z) of the EoS is obtained.

The van der Waals one-fluid mixing rules are used for the energy, $A$ and for the volume, $B$ parameters of the EoS

$$
\begin{gathered}
A=\sum_{i=1}^{n} \sum_{j=1}^{n} x_{i} x_{j} \sqrt{A_{i}} \sqrt{A_{j}}\left(1-k_{i j}\right), \\
B=\sum_{i=1}^{n} x_{i} B_{i}
\end{gathered}
$$

where $A_{i}=\Omega_{a i} p_{r i} / T_{r i}^{2}\left[1+m_{i}\left(\omega_{i}\right)\left(1-T_{r i}^{0.5}\right)\right]^{2}$ and $B_{i}=$ $\Omega_{b i} p_{r i} / T_{r i} . \Omega_{a}, \Omega_{b}$ and $m(\omega)$ are specific to each EoS.

The logarithm of equilibrium constants is

$$
\begin{aligned}
& \ln K_{i}=C_{0}+C_{1} \sqrt{A_{i}}+C_{2} B_{i}+\sum_{k=3}^{m+2} C_{k} \gamma_{k-2, i} ; \\
& \quad i=1, n,
\end{aligned}
$$

where

$$
\begin{gathered}
C_{0}=-\ln \left(Z_{L}-B_{L}\right)+\ln \left(Z_{V}-B_{V}\right), \\
C_{1 p}=\frac{2}{\left(\delta_{1}-\delta_{2}\right)} \frac{\lambda_{p}^{(i)}}{B_{p}} \cdot \ln \left(\frac{Z_{p}+\delta_{1} B_{p}}{Z_{p}+\delta_{2} B_{p}}\right) ; p=L, V,
\end{gathered}
$$

$$
\begin{gathered}
C_{1}=C_{1 V}-C_{1 L} \\
C_{2}=\frac{Z_{L}-1}{B_{L}}-\frac{Z_{V}-1}{B_{V}}-\frac{1}{2}\left(\frac{C_{1 L}}{\lambda_{L}^{(i)}} \frac{A_{L}}{B_{L}}-\frac{C_{1 V}}{\lambda_{V}^{(i)}} \frac{A_{V}}{B_{V}}\right)
\end{gathered}
$$

and

$$
C_{k}=\chi_{k}^{(i)}\left(\frac{C_{1 L}}{\lambda_{L}^{(i)}} \theta_{k L}-\frac{C_{1 V}}{\lambda_{V}^{(i)}} \theta_{k V}\right) ; k=3, m+2,
$$

where

$$
\begin{gathered}
A_{p}=\alpha_{p}^{2}+2 \sum_{k=1}^{m} \theta_{k p} \gamma_{k p}+\sum_{k=1}^{m} \theta_{k p}^{2} ; p=L, V, \\
\alpha=\sum_{j=m+1}^{n c} y_{j} \sqrt{A_{j}}, \\
\theta_{k}=y_{k} \sqrt{A_{k}} ; \quad k=1, m, \\
\gamma_{k}=\sum_{i=k+1}^{n} y_{i} \gamma_{k i} ; \quad k=1, m,
\end{gathered}
$$

$$
\begin{gathered}
\gamma_{k i}=\sqrt{A_{i}}\left(1-k_{k i}\right) ; \quad k=1, m ; \quad i=k+1, n, \\
\lambda^{(i)}=\left\{\begin{array}{c}
\theta_{i}+\gamma_{i} ; \quad i=1, m \\
\alpha \quad ; \quad i=m+1, n
\end{array}\right.
\end{gathered}
$$

and $\chi_{j}^{(i)}=0 ; i \leq j ; \chi_{j}^{(i)}=1 ; i>j$.

Finally, the elements of the reduction matrix in equation (6) are: $q_{0 i}=1, q_{1 i}=\sqrt{A_{i}}, \quad q_{2 i}=B_{i}, \quad$ and $q_{k i}=\gamma_{k i} ; k=3, m+2$, for $i=1, \ldots, n$. 